\title{
Observation of transient gain without population inversion in a laser-cooled rubidium $\Lambda$ system
}

\author{
S. R. de Echaniz, ${ }^{1}$ Andrew D. Greentree, ${ }^{1}$ A. V. Durrant, ${ }^{1}$ D. M. Segal, ${ }^{2}$ J. P. Marangos, ${ }^{2}$ and J. A. Vaccaro ${ }^{3}$ \\ ${ }^{1}$ Quantum Processes Group, Department of Physics and Astronomy, The Open University, Walton Hall, Milton Keynes MK7 6AA, \\ United Kingdom \\ ${ }^{2}$ Laser Optics and Spectroscopy Group, Blackett Laboratory, Imperial College of Science, Technology and Medicine, \\ Prince Consort Road, London SW7 2BW, United Kingdom \\ ${ }^{3}$ Department of Physics and Astronomy, University of Hertfordshire, College Lane, Hatfield AL10 9AB, United Kingdom
}

(Received 17 May 2001; published 10 October 2001)

\begin{abstract}
We have observed clear Rabi oscillations of a weak probe in a strongly driven three-level $\Lambda$ system in laser-cooled rubidium. When the coupling field is nonadiabatically switched on using a Pockels cell, transient probe gain without population inversion is obtained in the presence of uncoupled absorptions. Our results are supported by three-state computations.
\end{abstract}

DOI: 10.1103/PhysRevA.64.055801

PACS number(s): 42.50.Gy, 42.50.Md, 42.50.Hz, 32.80.Pj

In the past decade there has been growing interest in the study of atomic coherence effects in multilevel atoms, especially coherent population trapping [1], electromagnetically induced transparency (EIT) [2], slow light propagation [3], and optical gain without inversion. Reviews of lasing without inversion have been given by Kocharovska and Scully [4] and more recently by Mompart and Corbalán [5]. Most of this work has been in the steady-state regime and the fast picosecond pulsed regime [6], but there have been several studies of transient effects where the coherence is switched on or off rapidly in a time that is short compared to decay rates and Rabi frequencies, i.e., in the nonadiabatic regime. An early experiment by Fry et al. [7] demonstrated several transient effects in a three-level $\Lambda$ system realized in a sodium vapor cell. Their observations included transient gain of a strong field when a weak field was switched on by a Pockels cell in the presence of incoherent pumping to the upper level, but without population inversion. Subsequently, several theoretical studies of nondegenerate three-level lambda, V, and cascade systems have predicted transient ringing of a probe beam with gain when a strong coupling beam is switched on [8,9]. This predicted transient gain can occur without population inversion on the probe transition, and occurs with and without incoherent pumping. This ringing has also been explained by Vaccaro et al. [10] via stochastic wave-function diagrams. Transient ringing of a threelevel system with gain has been observed in the radiofrequency regime in nitrogen-vacancy centers in diamond samples [11] but not yet in the optical region. Since the ringing occurs at approximately the Rabi frequency of the strong field, experiments in the optical region would need to be carried out in a Doppler-free configuration or ideally in a laser-cooled sample to avoid the Doppler effects masking the coherent effects.

In our previous work [12] we observed the transient approach to EIT in a cold rubidium $\Lambda$ system in a magnetooptical trap (MOT). When the coupling field was switched on by a Pockels cell, the rapid rise in probe transparency exhibited an overshoot before settling down to the steady state. This overshoot was interpreted as the first Rabi halfcycle in the transient ringing, but it did not reach gain owing to absorption on uncoupled Zeeman transitions and twophoton dephasing effects. In the experiments reported in this paper the coupling field intensity has been increased to the point where a clear Rabi ringing cycle reaching well into gain is observed. The results are supported by density-matrix computations of a three-level $\Lambda$ system supplemented by observational data on the strengths of uncoupled absorptions and the effects of the MOT fields.

The $\Lambda$ system we have studied is formed by the weak probe field $P$ and the strong-coupling field $C$ shown in Fig. 1. The ${ }^{87} \mathrm{Rb}$ sample was cooled in a standard MOT with trapping field $T$ and repumping field $R$, similar to the one used in our previous work on EIT [13]. All laser fields were derived from external-cavity grating-controlled 780-nm laser diodes in master and master-slave arrangements, acting on the hyperfine transitions of the $5 S_{1 / 2}$ to $5 P_{3 / 2} D_{2}$ line, with the exception of the trap's repumping beam $R$, which is locked to the $5 S_{1 / 2}$ to $5 P_{1 / 2} D_{1}$ line at 795-nm. The frequency of each master laser is monitored by saturated absorption in a Rb cell at room temperature and can be locked via electronic feed-

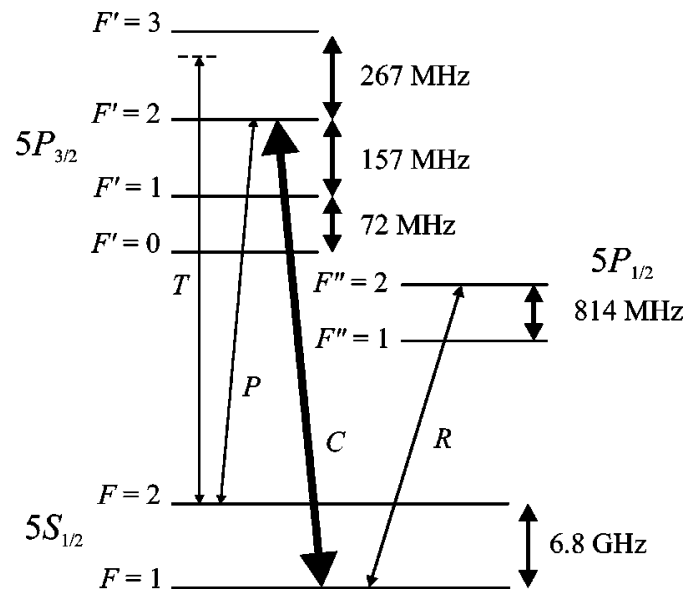

FIG. 1. The ${ }^{87} \mathrm{Rb}$ system in which the experiments are carried out. The atoms are trapped and cooled in a magneto-optical trap by trapping fields $T$ and $R$. The trapping beams are detuned by $-13 \mathrm{MHz}$ from the $F=2$ to $F^{\prime}=3$ transition of the $\mathrm{D}_{2}$ line. The probe beam $P$ and the coupling beam $C$ form a $\Lambda$ EIT system. 


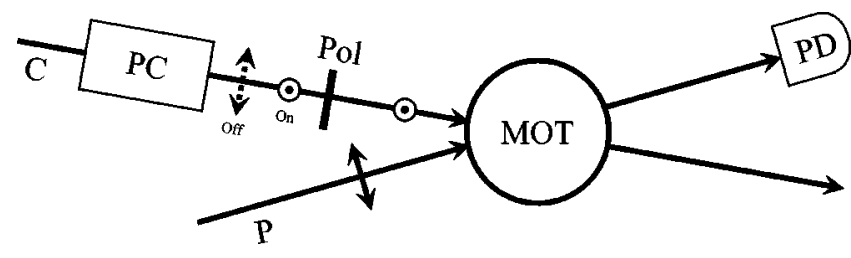

FIG. 2. Schematic of the experimental arrangement showing the relevant beams and their polarizations. Field $P$ is linearly polarized in the horizontal plane while field $C$ is linearly polarized in the vertical plane when the Pockels cell is on, or in the horizontal plane when the Pockels cell is off. The angle between fields $C$ and $P$ is about $20^{\circ}$. PD is an avalanche photodiode, PC is a Pockels cell, Pol is a polarizer. The trapping and repumping fields are not shown for clarity. back. In all master-slave arrangements, there is an acoustooptic modulator which shifts the frequency of the slave relative to the frequency of the master.

The trapping lasers $T$ are derived from a master-slave system. They are locked and detuned by $-13 \mathrm{MHz}$ from the $F=2$ to $F^{\prime}=3$ transition. Their diameter is $\approx 1 \mathrm{~cm}$ and the total average intensity in the cold sample is $\approx 50 \mathrm{~mW} / \mathrm{cm}^{2}$. The repumping beam $R$ is locked to the $F=1$ to $F^{\prime \prime}=2$ transition and has a diameter of $\approx 1 \mathrm{~cm}$ and an intensity of $\approx 0.2 \mathrm{~mW} / \mathrm{cm}^{2}$. The probe beam $P$ can be locked to or scanned across the $F=2$ to $F^{\prime}=2$ transition by piezocontrol of the external cavity. $P$ has an average intensity $\approx 0.03 \mathrm{~mW} / \mathrm{cm}^{2}$ in a diameter $\approx 1 \mathrm{~mm}$. The coupling beam $C$ is locked on resonance with the $F=1$ to $F^{\prime}=2$ transition (a)

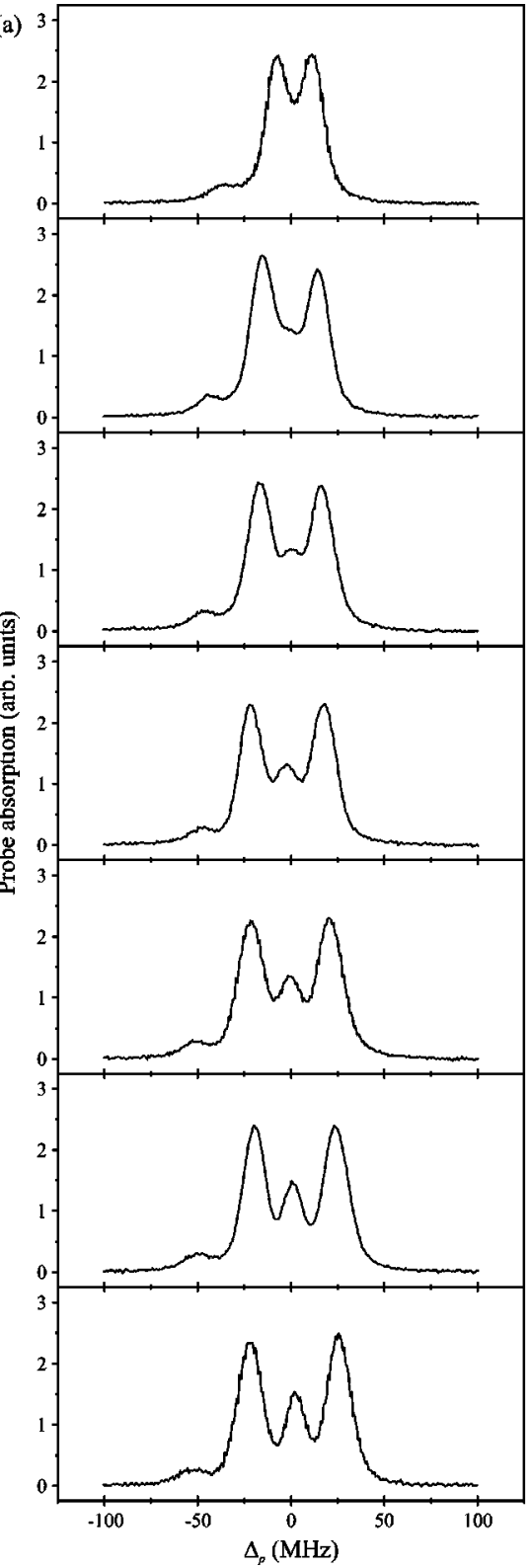

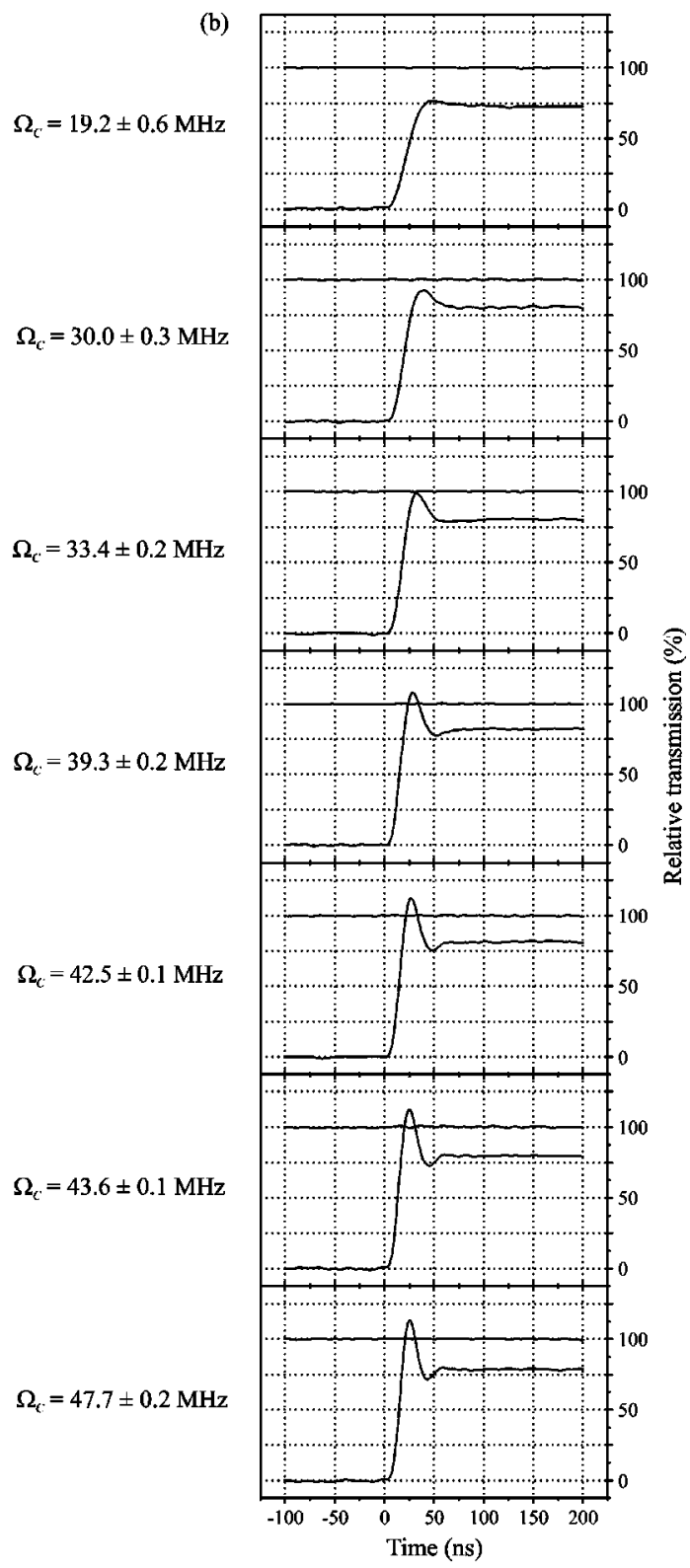

FIG. 3. (a) Steady-state probe absorption spectra with $C$ on and locked to the light-shifted transition of the atoms in the MOT. (b) Probe transient transmission as $C$ is turned on at time $t=0$. Each trace is an average over 200 scans. 


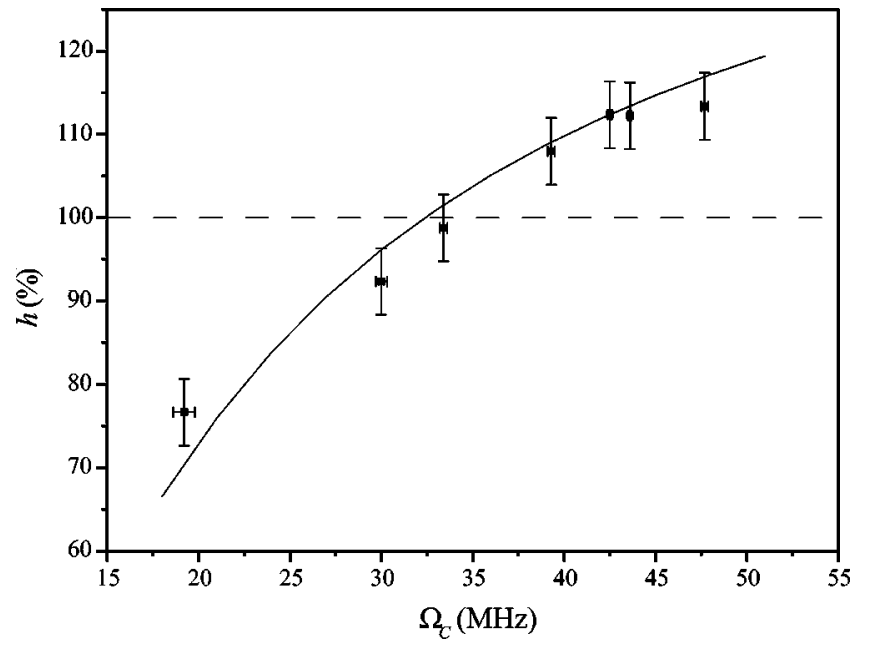

FIG. 4. The height $h$ of the first Rabi cycle peak plotted against $\Omega_{C}$ when $C$ is locked to the light-shifted transition of the atoms in the MOT. The solid line is a theoretical model.

with an average intensity of $\approx 100 \mathrm{~mW} / \mathrm{cm}^{2}$ in a roughly elliptical profile $2 \mathrm{~mm} \times 4 \mathrm{~mm}$.

The experimental setup is shown schematically in Fig. 2. The probe $P$ is linearly polarized in the horizontal plane while the coupling field $C$ is linearly polarized in the vertical direction. The probe propagates at an angle of about $20^{\circ}$ with respect to $C$, which was found to give a good overlap of the probe with the coupling field in the cold sample. It can be shown [14] that this arrangement of polarizations is equivalent to three separate sets of Zeeman levels in $\Lambda$ configurations plus Zeeman transitions of the probe uncoupled by $C$. A Pockels cell and a polarizer are placed in the path of $C$ so that this beam can be switched on and off.

Figure 3(a) shows the steady-state probe absorption versus probe detuning $\Delta_{p}$ for different coupling field Rabi frequencies as the probe beam is scanned across the $F=2$ to $F^{\prime}=2$ transition. It is seen that the spectrum consists of a central peak situated between the two Autler-Townes peaks of a standard EIT profile and a small peak to its red side. The central peak in the spectrum is caused by $P$ probing Zeeman sublevels that are not coupled by $C$. We identified this peak as the uncoupled absorption peak in our previous work [14]. The trapping beams form a V-type EIT system with $P$, splitting each peak in two, which gives rise to the small reddetuned peak. We note that, in the present work, it was necessary to shift the frequencies of $C$ and $P$ to take account of the light shifts caused by the strong-coupling field $C$ and the trapping beams $T$ as described in [14]. The shifts of $C$ varied with $\Omega_{C}$ with a maximum value of $-7 \mathrm{MHz}$, while the shift of the probe was $4 \mathrm{MHz}$. Finally, we note that the linewidths of our spectra are well modeled by inclusion of the broadening due to beam-profile inhomogeneities, variations of Clebsch-Gordan coefficients between different Zeeman transitions, and the spread of intensity in the standing wave field of the counterpropagating trapping beams in the MOT, which causes a spread in the detuning of the probe field due to the spatially varying light shifts.

The transient experiments were performed with the probe field locked to the $F=2$ to $F^{\prime}=2$ transition of the cold sample and nonadiabatically turning on the coupling field in less than $6 \mathrm{~ns}$ using a Pockels cell driven by a pulse $10 \mu \mathrm{s}$ long with a repetition rate of $10 \mathrm{~Hz}$. As the Pockels cell is switched on, it rotates the polarization of the coupling beam, which then passes through a polarizer that selects only the rotated polarization (see Fig. 2).

The steady-state probe absorption without the coupling field is $\approx 20 \%$. Figure $3(\mathrm{~b})$ shows the probe transmission relative to this initial value, taken to be $0 \%$, when $C$ is turned on at time $t=0$ for various coupling field Rabi frequencies $\Omega_{C}$. It is to be noted that the probe transmission relaxes approximately to the $80 \%$ level instead of the $100 \%$ level as predicted by $[8,9]$. This is mainly due to the uncoupled absorption peak and, to a lesser degree, dephasing mechanisms and inhomogeneities which reduce the visibility of the transparency window, as seen in the respective steady-state EIT traces in Fig. 3(a). Another important feature of these traces is the fact that only one whole Rabi cycle is seen, mainly because of the line broadening caused by the spread in trapping beam intensities mentioned above. This is in good agreement with our model when the spread in trapping beam intensity is taken into account.

Despite the above two limiting effects, it is possible to see as much as $15 \pm 5 \%$ gain for maximum $\Omega_{C}$, and an increase in the frequency of the Rabi cycles with $\Omega_{C}$ as predicted by the theory. Figure 4 shows the different heights $h$ of the first Rabi cycle as a function of $\Omega_{C}$. Gain is seen in this plot after a threshold Rabi frequency of $\Omega_{C} \approx 33 \mathrm{MHz}$. Numerically subtracting the effects of the uncoupled absorptions would yield a maximum gain $\approx 45 \%$ and a significantly lower threshold Rabi frequency. The solid line in Fig. 4 corresponds to a theoretical expectation derived from a three-level atom model with an initial population distribution of $75 \%$ in the $F=2$ and $25 \%$ in the $F=1$ ground levels, as established from independent absorption measurements. Our computations show that for these initial conditions there is no inversion at any time on either the one-photon probe transition or the two-photon transition $F=1 \rightarrow F^{\prime}=2 \rightarrow F=2$.

Theoretical modeling of our system was performed by numerically integrating the density-matrix equations of motion presented earlier [8] and incorporating the spread of probe detunings due to the spatial variation in the light shifts induced by trapping fields. It is difficult to fully model this spread in trapping field intensity as the standing-wave pattern is critically dependent on the slowly varying, but unknown, relative phases of the component fields of the trapping beams. For a fuller description of these effects, see [15]. Experimentally, the mean light shift was corrected by shifting the frequency of the probe field accordingly, while in the numerical models, a truncated Lorentzian distribution of detuned probe fields was found to give good agreement with the experimental results. More accurate modeling of the actual trap configuration should, however, yield better fits to the data.

We have carried out experiments showing transient ringing of a three-level $\Lambda$ system in laser-cooled rubidium when the coupling field is nonadiabatically turned on. We have observed as much as $15 \pm 5 \%$ transient gain in the presence 
of uncoupled absorption. We note that by working with other configurations, for example by reversing the roles of $C$ and $P$ in a dark SPOT trap [16], it should be possible to eliminate the broadening effects of the trapping fields and the uncoupled absorptions, thereby to observe a larger gain.
We would like to thank the EPSRC for financial support on this project and Dr. T. B. Smith (Open University) for useful discussions. We would also like to thank Roger Bence, Fraser Robertson, and Robert Seaton (Open University) and Shahid Hanif (Imperial College) for technical assistance.
[1] H.Y. Ling, Y-Q. Li, and M. Xiao, Phys. Rev. A 53, 1014 (1996); E. Arimondo, Prog. Opt. 35, 257 (1996).

[2] S.E. Harris, Phys. Today 50(7), 36 (1997); J.P. Marangos, J. Mod. Opt. 45, 471 (1998); M. Xiao, Y-Q. Li, S-Z. Jin, and J. Gea-Banacloche, Phys. Rev. Lett. 74, 666 (1995).

[3] M.M. Kash, V.A. Sautenkov, A.S. Zibrov, L. Hollberg, G.R. Welch, M.D. Lukin, Y. Rostovtsev, E.S. Fry, and M.O. Scully, Phys. Rev. Lett. 82, 5229 (1999); L.V. Hau, S.E. Harris, Z. Dutton, and C.H. Behroozi, Nature (London) 397, 594 (1999).

[4] O. Kocharovskaya, Phys. Rep. 219, 175 (1992); M.O. Scully, ibid. 219, 191 (1992).

[5] J. Mompart and R. Corbalán, J. Opt. B: Quantum Semiclassical Opt. 2, R7 (2000).

[6] A. Nottelmann, C. Peters, and W. Lange, Phys. Rev. Lett. 70, 1783 (1993).

[7] E.S. Fry, X. Li, D. Nikonov, G.G. Padmabandu, M.O. Scully, A.V. Smith, F.K. Tittel, C. Wang, S.R. Wilkinson, and S-Y. Zhu, Phys. Rev. Lett. 70, 3235 (1993).

[8] Y-Q. Li and M. Xiao, Opt. Lett. 20, 1489 (1995); Y. Zhu, Phys.
Rev. A 55, 4568 (1997).

[9] Y. Zhu, Phys. Rev. A 53, 2742 (1996); J. Mompart, C. Peters, and R. Corbalán, Quantum Semiclassic. Opt. 10, 355 (1998).

[10] J.A. Vaccaro, A.V. Durrant, D. Richards, S.A. Hopkins, H.X. Chen, and K.E. Hill, J. Mod. Opt. 45, 315 (1998).

[11] C. Wei, N.B. Manson, and J.P.D. Martin, Phys. Rev. Lett. 74, 1083 (1995).

[12] H.X. Chen, A.V. Durrant, J.P. Marangos, and J.A. Vaccaro, Phys. Rev. A 58, 1545 (1998).

[13] S.A. Hopkins, E. Usadi, H.X. Chen, and A.V. Durrant, Opt. Commun. 138, 185 (1997).

[14] S. R. de Echaniz, A. D. Greentree, A. V. Durrant, D. M. Segal, J. P. Marangos, and J. A. Vaccaro, Phys. Rev. A 64, 013812 (2001); e-print quant-ph/0102098.

[15] S.A. Hopkins and A.V. Durrant, Phys. Rev. A 56, 4012 (1997); S. A. Hopkins, Ph.D. thesis, The Open University, 1995.

[16] W. Ketterle, K.B. Davis, M.A. Joffe, A. Martin, and D.E. Pritchard, Phys. Rev. Lett. 70, 2253 (1993). 